\title{
In vivo Electrophysiological Evidence for the Regulatory Role of Autoreceptors on Serotonergic Terminals
}

\author{
Yves Chaput, Pierre Blier, and Claude de Montigny \\ Neuroscience Research Center, Department of Physiology, Faculty of Medicine, University of Montreal, Montreal, \\ Quebec H3C 3J7, Canada
}

\begin{abstract}
The present in vivo studies were undertaken to evaluate electrophysiologically the modulatory role of the terminal 5-HT autoreceptor on 5-HT neurotransmission. In a first series of experiments, the effect of the electrical activation of the ascending 5-HT pathway on the firing activity of CA3 hippocampal pyramidal neurons was measured before and after the intravenous administration of methiothepin, a terminal 5-HT autoreceptor antagonist. Methiothepin significantly increased the duration of the suppression of firing activity of these neurons by the electrical stimulation of the 5-HT pathway, without modifying their responsiveness to microiontophoretically applied 5-HT. This suggests that endogenously released 5-HT activates the 5HT terminal autoreceptor and that methiothepin enhances the efficacy of 5-HT synaptic transmission by blocking this activation. In a second series of experiments, further evidence for the activation of terminal 5-HT autoreceptors by 5-HT released by the electrical stimulation was sought by assessing the effectiveness of 2 series of stimulations of the ascending 5-HT pathway delivered at different frequencies while recording the same postsynaptic neuron. Increasing the frequency of stimulation (from 0.8 to $5 \mathrm{~Hz}$ ) significantly reduced the duration of suppression of firing activity of the postsynaptic neurons. This difference between the 0.8 and $5 \mathrm{~Hz}$ stimulations was decreased by intravenous methiothepin, suggesting that the reduced effectiveness of the stimulations delivered at the higher frequency is attributable to a greater activation of the terminal 5-HT autoreceptor. These results provide direct electrophysiological evidence for the modulatory role of the 5-HT terminal autoreceptor on 5-HT neurotransmission.
\end{abstract}

Most of the evidence supporting the existence of autoreceptors on 5-HT terminals has been derived from in vitro data on the release of 5-HT from rat brain synaptosomes and slices (for a review, see Moret, 1985). These studies have consistently demonstrated that exogenously applied agonists such as 5-HT itself and lysergic acid diethylamide (LSD) decrease the electrically or $\mathrm{K}^{+}$-evoked release of ${ }^{3} \mathrm{H}-5-\mathrm{HT}$ in a concentration-dependent manner (Baumann and Waldmeier, 1981; Cerrito and Raiteri, 1979; Chase et al., 1969; Cox and Ennis, 1982; Farnebo and Hamberger, 1971, 1974; Göthert, 1980; Göthert and Weinheimer, 1979; Hamon et al., 1974; Katz and Kopin, 1969; Langer and Moret, 1982; Martin and Sanders-Bush, 1982; Mounsey et al., 1982). In such preparations, the antagonist property of meth-

Received Aug. 19, 1985; revised Jan. 27, 1986; accepted Mar. 24, 1986.

This work was supported in part by Medical Research Council (MRC) of Canada Grant MA-6444 (C. de M.), by a Fellowship (Y.C.), and a Studentship (P.B.) from the Fonds de la Recherche en Santé du Québec, and, by a MRC Scientist Award (C. de M.). We thank L. Perrault for typing the manuscript, G. F. Filosi and E. Rupnik for preparing the illustrations, and L. Caille for writing the computer programs.

Correspondence should be addressed to Yves Chaput at the above address.

Copyright (C) 1986 Society for Neuroscience $0270-6474 / 86 / 102796-06 \$ 02.00 / 0$ iothepin has been demonstrated by its ability to reduce the effect of exogenous 5-HT and LSD on the depolarization-induced ${ }^{3} \mathrm{H}$ 5-HT release; the parallel rightward shift of the dose-response curves for the inhibition of ${ }^{3} \mathrm{H}-\mathrm{HT}$ release by 5 -HT and LSD suggests that methiothepin prevents the effect of these agonists by a steric interaction at the autoreceptor site (Cerrito and Raiteri, 1979; Cox and Ennis, 1982; Farnebo and Hamberger, 1974; Göthert, 1980; Langer and Moret, 1982). Methiothepin by itself increases the electrically or $\mathrm{K}^{+}$-evoked release of ${ }^{3} \mathrm{H}-5-\mathrm{HT}$, suggesting that it antagonizes the effect of endogenously released 5-HT on the terminal 5-HT autoreceptor (Cerrito and Raiteri, 1979; Göthert, 1980; Langer and Moret, 1982). Furthermore, Göthert (1980) and Baumann and Waldmeier (1981) observed that increasing the frequency of electrical stimulation decreased the overflow of ${ }^{3} \mathrm{H}-5-\mathrm{HT}$ released per stimulation, presumably due to a greater activation of the terminal autoreceptor by endogenous 5-HT.

To date, in vivo evidence in support of the above in vitro data has been sparse. Baumann and Waldmeier (1984) performed differential pulse voltammetry in unanesthetized rats in order to detect what is thought to be extraneuronal 5-hydroxyindoleacetic acid (5-HIAA) following the intraperitoneal administration of various terminal 5-HT autoreceptor agonists and antagonists. Consistent with its potent terminal 5-HT autoreceptor blocking property in vitro, methiothepin increased the 5-HIAA signal in the 3 regions studied (hippocampus, hypothalamus, and corpus striatum). Interestingly, LSD, even when administered at relatively high doses, produced only a minimal decrease of the 5-HIAA signal in the 2 brain regions studied (hippocampus and hypothalamus). Petitbone and Pflueger (1984), measuring 5-hydroxytryptophan accumulation following aromatic amino acid decarboxylase inhibition in the rat hypothalamus, demonstrated a significant reduction in its accumulation following the intraperitoneal administration of LSD; this effect was antagonized by a pretreatment with methiothepin at a dose that, by itself, did not alter 5-hydroxytryptophan accumulation. Whether these effects of LSD and methiothepin result from the activation and blockade of the somatic or terminal 5-HT autoreceptor is unclear, since these agents are active not only at the terminal autoreceptor but also at the somatic autoreceptor (Aghajanian et al., 1972; Gallager and Aghajanian, 1975; Rebec et al., 1982).

The present electrophysiological studies were undertaken to assess in vivo the functional role of the terminal 5-HT autoreceptor, by measuring the effectiveness of the electrical activation of the ascending 5-HT pathway on the firing activity of the postsynaptic target neurons.

\section{Materials and Methods}

Male Sprague-Dawley rats (240-270 g) were anesthetized with chloral hydrate $(400 \mathrm{mg} / \mathrm{kg}$, i.p.) and mounted in a stereotaxic apparatus. Fivebarrelled glass micropipettes were prepared and their tips broken back 


\section{A PRIOR TO METHIOTHEPIN}

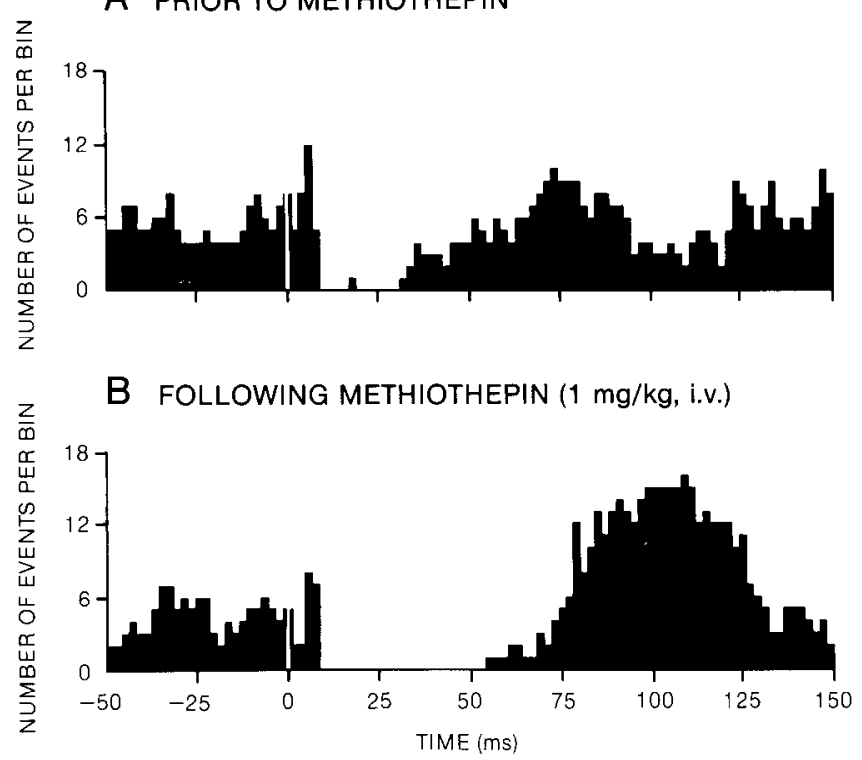

Figure 1. Peristimulus time histograms obtained from the same CA3 hippocampal pyramidal neuron before $(A)$ and after $(B)$ the intravenous administration of methiothepin $(1 \mathrm{mg} / \mathrm{kg})$. Each histogram was constructed from 200 stimuli of $0.5 \mathrm{msec}$ delivered at $0.8 \mathrm{~Hz}$ with an intensity of $320 \mu \mathrm{A}$. Bin width, $2 \mathrm{msec}$. Stimulation pulse was delivered at time 0 .

to 10-12 $\mu \mathrm{m}$ under microscopic control (Haigler and Aghajanian, 1974). The central barrel, filled with a $2 \mathrm{~m} \mathrm{NaCl}$ solution saturated with Fast green FCF, was used for extracellular unitary recording. The side barrels contained the following solutions: 5 -HT creatinine sulfate $0.5 \mathrm{~mm}$ in $0.2 \mathrm{M} \mathrm{NaCl}, \mathrm{pH} 4$; Sigma), norepinephrine bitartrate (NE; $0.2 \mathrm{M}$ in 0.2 $\mathrm{M} \mathrm{NaCl}, \mathrm{pH} 4$; Sigma), acetylcholine chloride ( $\mathrm{ACh} ; 20 \mathrm{~mm}$ in $0.2 \mathrm{M}$ $\mathrm{NaCl}, \mathrm{pH} 4$; Sigma), and $\mathrm{NaCl} 2 \mathrm{M}$, the latter barrel being used for automatic current balancing. The microelectrode was descended at 4.2 $\mathrm{mm}$ lateral and $4.2 \mathrm{~mm}$ anterior to lambda into the CA3 region of the dorsal hippocampus. CA3 hippocampal pyramidal neurons were identified by their large-amplitude $(0.5-1.2 \mathrm{mV})$ and long-duration $(0.8-1.2$ msec) action potentials and by their characteristic complex spike discharge alternating with simple spike activity (Kandel and Spencer, 1961). Fast green was deposited at the last recording site with a $-26 \mu \mathrm{A}$ current for subsequent histological verification. Since most hippocampal pyramidal neurons are not spontaneously active in chloral hydrate-anesthetized rats, a small current of ACh (between 2 and $5 \mathrm{nA}$ ) was used to maintain a firing rate in the physiological range $(8-12 \mathrm{~Hz})$. Neuronal responsiveness to microiontophoretic applications of 5-HT and NE was assessed in 2 ways: (1) using the $\mathrm{I} \cdot \mathrm{T}_{50}$ method, which, in brief, consists of determining the charge [current (in $\mathrm{nA}) \times$ time (in sec)] required to obtain a $50 \%$ depression of firing rate (de Montigny and Aghajanian, 1977); and (2) the number of spikes suppressed per $n \mathrm{~A}$, which is obtained by dividing the total number of spikes suppressed from the beginning of the ejection period, up to a recuperation of $85 \%$ of the pre-ejection value, by the ejection current used (in $n \Lambda$ ). The $I \cdot T_{50}$ value, which derives from the rate of onset of the effect, is believed to assess primarily the sensitivity of the postsynaptic neurons (de Montigny and Aghajanian, 1977; Wang et al., 1979), whereas the total number of spikes suppressed measures the global efficacy of the microiontophoretic application.

In order to activate the ascending 5-HT pathway, a bipolar concentric electrode (NE-100, David Kopf Instruments) was positioned on the midline at a backward angle of $10^{\circ}$ in the ventromedial tegmentum (VMT) at $\mathbf{P}=5.8, \mathrm{~V}=8$ according to the atlas of Paxinos and Watson (1982). At the end of each experiment, a current of $0.5 \mathrm{~mA}$ was passed for $8 \mathrm{sec}$ through the stimulating electrode to produce a lesion for subsequent histological verification of the location of the electrode tip. Square pulses of $0.5 \mathrm{msec}$ were delivered at $0.2,0.8$, and $5.0 \mathrm{~Hz}$ with intensities of $80,160,320$, and $640 \mu \mathrm{A}$. Pulses were generated by an S8 stimulator (Grass) and were delivered through a direct-coupled isolation unit (SIV 478A, Grass). The unitary activity was recorded on

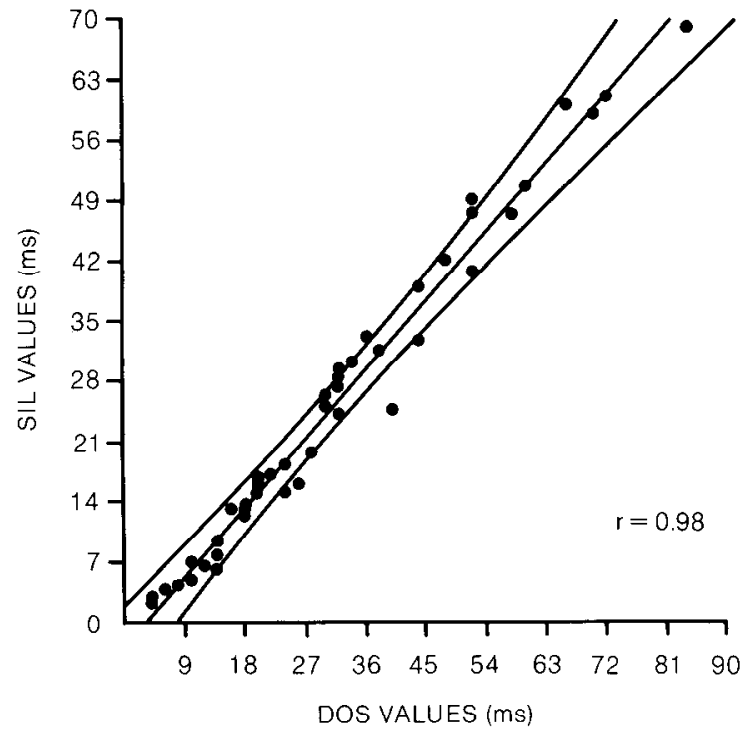

Figure 2. Analysis of regression showing the relationship between the DOS value and its corresponding SIL value calculated from the response of a CA3 hippocampal pyramidal neuron to the electrical stimulation of the ascending 5-HT pathway. Since the SIL value is derived from the DOS value by computer, only DOS values greater than $0 \mathrm{msec}$ were used $(n=49)$. Outer curves represent the SD. $p<0.001$.

magnetic tapes and subsequently analyzed on an IBM PC computer equipped with a Tecmar interface. Peristimulus time histograms of hippocampal pyramidal neuron firing activity were generated to measure the suppression of firing. Two parameters were used for this purpose: (1) the duration of suppression of firing (DOS, in msec), defined as the time interval initiated by a $50 \%$ reduction in the number of events per bin from the mean prestimulation probability of firing, to the time it returned to $90 \%$ of that same prestimulation value; and (2) the absolute silence (SIL, in $\mathrm{msec}$ ), defined as the theoretical duration of a total suppression of firing that would represent the number of events suppressed by the stimulation. This latter value was calculated by computer by dividing the total number of events missing after the stimulation by the mean prestimulation frequency of firing. Thus, the SIL value corresponds to the DOS value normalized for the firing activity of the neuron recorded.

The results presented below derive from SIL and DOS values obtained with 2 series of stimulations of the same intensity while recording from the same postsynaptic neuron. In the pharmacological experiments, most stimulations were carried out at a frequency of $0.8 \mathrm{~Hz}$. SIL and DOS values were obtained from a first series of stimulations prior to drug administration $\left(S_{1}\right)$ and from a second series $\left(S_{2}\right)$ following drug administration. In the case of methiothepin, $S_{2}$ was begun 5 min after its intravenous administration, while it was started $1-5 \mathrm{~min}$ after the intravenous administration of LSD. In the experiments in which the effect of the frequency of stimulation was examined, the same number of pulses was delivered for each of the 2 stimulation periods $\left(S_{1}\right.$ and $\mathrm{S}_{2}$ ), the intensity of the stimulation being kept constant for any given neuron. Hence, all data were generated in pairs and analyzed for statistical significance using the paired Student's $t$ test.

\section{Results}

The electrical activation of the ascending 5-HT pathway at a frequency of $0.8 \mathrm{~Hz}$ resulted in a mean DOS of hippocampal pyramidal neuron firing of $16 \pm 2.5 \mathrm{msec}(n=30)$ and $34 \pm$ $3.2 \mathrm{msec}(n=43)$ at 160 and $320 \mu \mathrm{A}$, respectively (Fig. $1 A)$, with a mean latency from the delivery of the stimulation to the onset of suppression of $5.6 \pm 0.4 \mathrm{msec}$. There was no correlation between DOS values and ejection currents of ACh used $(r=$ $0.003 ; n=80)$. The corresponding SIL values were $13 \pm 2.0$ $\operatorname{msec}(n=30)$ and $29 \pm 2.5 \mathrm{msec}(n=43)$ at 160 and $320 \mu \mathrm{A}$, respectively. Since DOS and SIL values were found to be highly correlated ( $r=0.97$; Fig. 2 ), only SIL values are used below for 


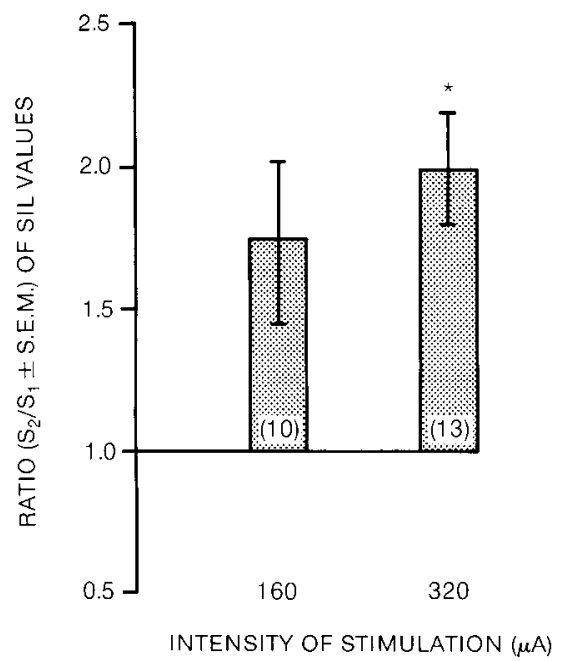

Figure 3. $\mathrm{S}_{2} / \mathrm{S}_{1}$ ratios of the SIL values of CA3 hippocampal pyramidal neurons obtained with the stimulation of the ascending 5-HT pathway at intensities of 160 and $320 \mu \mathrm{A}$ before $\left(S_{1}\right)$ and following $\left(S_{2}\right)$ the intravenous administration of methiothepin. Methiothepin was administered at a dose of $1 \mathrm{mg} / \mathrm{kg}(160 \mu \mathrm{A}, n=9 ; 320 \mu \mathrm{A}, n=8), 0.5 \mathrm{mg} /$ $\mathrm{kg}(320 \mu \mathrm{A}, n=4)$, and $0.25 \mathrm{mg} / \mathrm{kg}(160 \mu \mathrm{A}, n=1 ; 320 \mu \mathrm{A}, n=1)$. The number of neurons tested is in parentheses. ${ }^{*} p<0.001$, using paired Student's $t$ test.

presenting the results of the pharmacological and frequency experiments. Only 3 neurons failed to respond to $320 \mu \mathrm{A}$ stimulation and required $640 \mu \mathrm{A}$ to show a detectable suppression of their firing activity. The $80 \mu \mathrm{A}$ stimulation produced a detectable suppression of firing in only 3 of 14 neurons tested with this current. Therefore, only data obtained with stimulations of 160 and $320 \mu \mathrm{A}$ have been used for statistical analysis.

In order to assess the net effect of methiothepin on the efficacy of 5-HT synaptic transmission, a single dose per rat was administered intravenously. In 17 experiments, a $1 \mathrm{mg} / \mathrm{kg}$ dose of methiothepin was administered; in 6 others, a lower dose, 0.25 or $0.5 \mathrm{mg} / \mathrm{kg}$, was used. At both the 160 and the $320 \mu \mathrm{A}$ intensities, methiothepin enhanced the effect of the stimulation (Figs. $1,3)$. The SIL values were increased to the same extent by the 3 doses of methiothepin administered, suggesting that maximal terminal 5-HT autoreceptor blockade was obtained at all 3 doses.

To determine the effect of acute methiothepin on the responsiveness of the postsynaptic hippocampal pyramidal neurons to $5-\mathrm{HT}$ and $\mathrm{NE}, \mathrm{I} \cdot \mathrm{T}_{50}$ values and the number of spikes suppressed

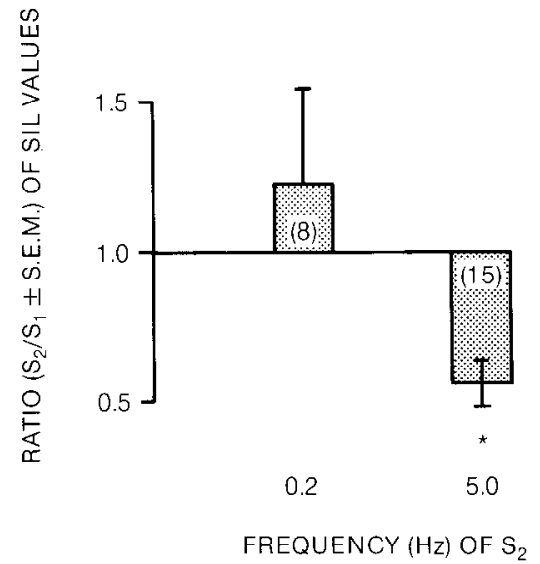

Figure 4. $\mathrm{S}_{2} / \mathrm{S}_{1}$ ratios of the SIL values of CA3 hippocampal pyramidal neurons obtained with the stimulation of the ascending 5-HT pathway at frequencies of $0.8\left(\mathrm{~S}_{1}\right)$ and $0.2\left(\mathrm{~S}_{2}\right) \mathrm{Hz}$ and at frequencies of $0.8\left(\mathrm{~S}_{1}\right)$ and $5.0\left(\mathrm{~S}_{2}\right) \mathrm{Hz}$. The number of neurons tested is indicated in parentheses. The intensity of the stimulations was $320 \mu \mathrm{A} .{ }^{*} p<0.001$, using paired Student's $t$ test.

per $\mathrm{nA}$ for both neurotransmitters were calculated before and after its administration. Methiothepin significantly altered both parameters for NE applications, indicating a decreased effectiveness of the neurotransmitter, but left unmodified those for 5-HT applications (Table 1).

In an attempt to detect the effect of 5-HT terminal autoreceptor activation by the electrically evoked release of endogenous 5-HT, we compared the effectiveness of 2 series of stimulations delivered at different frequencies while recording from the same postsynaptic neuron. This procedure was based on the assumption that the higher the frequency the greater should be the degree of activation of the terminal autoreceptor at the time of arrival of the next stimulation pulse. Therefore, a higher frequency of stimulation would be expected to produce a smaller effect on the postsynaptic neurons, each pulse liberating a smaller amount of 5-HT. In the first series of experiments, CA3 pyramidal neurons were recorded while stimulating the afferent $5-\mathrm{HT}$ axons at $0.8 \mathrm{~Hz}\left(\mathrm{~S}_{1}\right)$ and at $0.2 \mathrm{~Hz}\left(\mathrm{~S}_{2}\right)$. No statistically significant difference of the mean SIL values was found between the effects of the 2 stimulations, although there was a tendency towards an increase in the SIL value at the lower frequency (Fig. 4). In a second series of experiments, the effect of $0.8 \mathrm{~Hz}\left(\mathrm{~S}_{1}\right)$ and of $5 \mathrm{~Hz}\left(\mathrm{~S}_{2}\right)$ stimulations on the same neurons were compared. Stimulating the ascending 5-HT pathway with a fre-

Table 1. Responsiveness of CA3 hippocampal pyramidal neurons to microiontophoretic applications of 5-HT and NE before and after intravenous administration of methiothepin

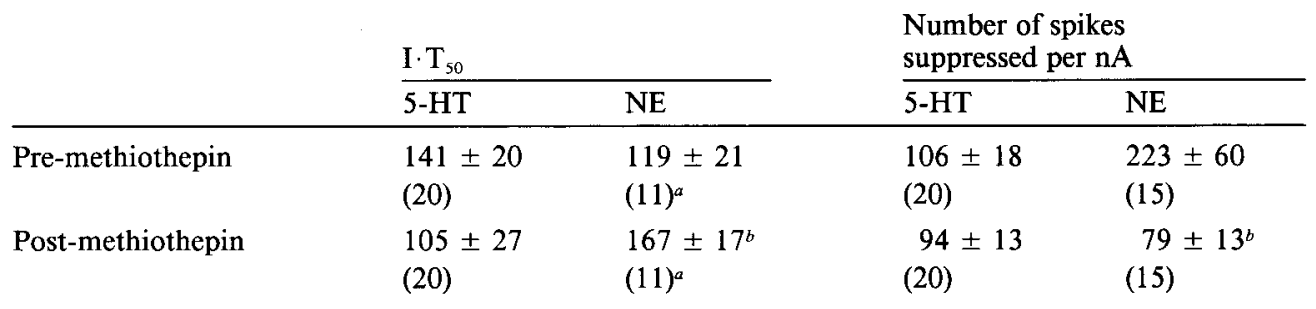

Values are expressed as means \pm SEM; responsiveness to 5-HT was assessed following doses of methiothepin of 1.0 $\mathrm{mg} / \mathrm{kg}(n=16)$ and $0.5 \mathrm{mg} / \mathrm{kg}(n=4)$ and to NE following doses of $1.0 \mathrm{mg} / \mathrm{kg}(n=14)$ and $0.5 \mathrm{mg} / \mathrm{kg}(n=1) ;$ numbers in parentheses indicate the number of neurons tested.

"In 4 neurons of the 15 neurons tested with NE, a $50 \%$ inhibition could not be obtained following methiothepin administration.

${ }^{b} p<0.05$ (compared to corresponding pre-drug values, using paired Student's $t$ test). 


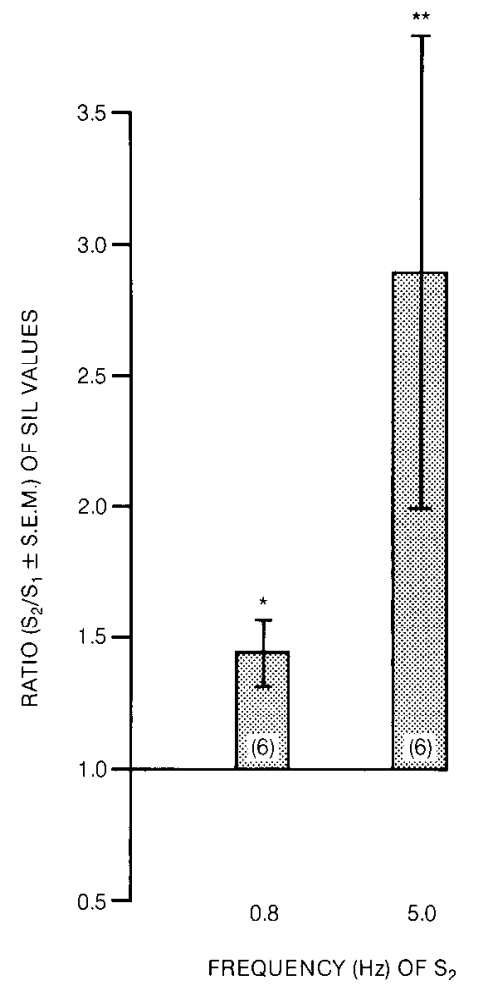

Figure 5. Graph showing the effect of the intravenous administration of methiothepin $(1 \mathrm{mg} / \mathrm{kg})$ on the $\mathrm{S}_{2} / \mathrm{S}_{1}$ ratios of the SIL values of CA3 hippocampal pyramidal neurons obtained with the stimulation of the ascending 5-HT pathway at frequencies of $0.8 \mathrm{~Hz}\left(\mathrm{~S}_{1}, \mathrm{~S}_{2}\right)$ and of $5.0 \mathrm{~Hz}$ $\left(S_{1}, S_{2}\right)$. The number of neurons tested is indicated in parentheses. The intensity of the stimulations was $320 \mu \mathrm{A} .{ }^{*} p<0.05,{ }^{* *} p<0.01$, using paired Student's $t$ test.

quency of $5 \mathrm{~Hz}$ markedly reduced the SIL value of the postsynaptic target neurons, as compared to the $S_{1}$ stimulation delivered at $0.8 \mathrm{~Hz}$ (Fig. 4).

To determine whether the difference between the SIL values produced by increasing the frequency of the stimulations could be reduced by a terminal 5-HT autoreceptor blockade, the response of the same postsynaptic neuron to the electrical stimulation of the ascending $5-\mathrm{HT}$ pathway at $0.8 \mathrm{~Hz}$ and at $5 \mathrm{~Hz}$ was assessed before $\left(S_{1}\right)$ and after $\left(S_{2}\right)$ the intravenous administration of methiothepin $(1 \mathrm{mg} / \mathrm{kg})$. Methiothepin produced a much greater effect on the $5 \mathrm{~Hz}$ than on the $0.8 \mathrm{~Hz}$ stimulations (Fig. 5). Although the mean post-methiothepin SIL value at 5 $\mathrm{Hz}$ was still lower than that at $0.8 \mathrm{~Hz}(46 \pm 6$ vs $56 \pm 8 \mathrm{msec}$, respectively; $n=6$ ), this difference was no longer statistically significant.

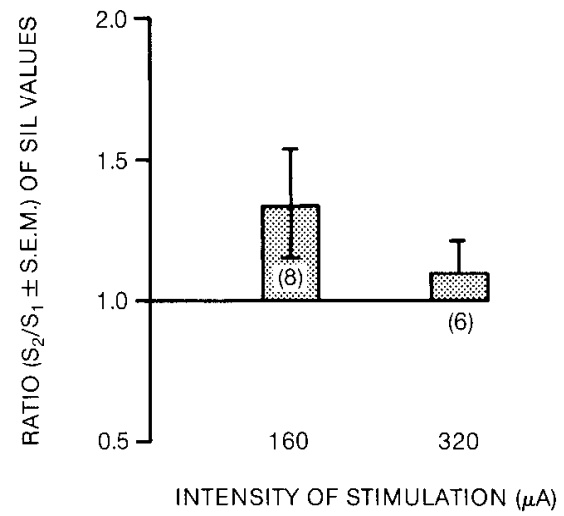

Figure 6. $\mathrm{S}_{2} / \mathrm{S}_{1}$ ratios of the SIL values of CA3 hippocampal pyramidal neurons obtained with the stimulation of the ascending 5-HT pathway at intensities of 160 and $320 \mu \mathrm{A}$ before $\left(\mathbf{S}_{1}\right)$ and following $\left(S_{2}\right)$ the intravenous administration of LSD, $25 \mu \mathrm{g} / \mathrm{kg}$. The effect of LSD was not statistically significant at either intensities of stimulations (paired Student's $t$ test).

Since the effect of methiothepin in our paradigm was more pronounced with the $5 \mathrm{~Hz}$ stimulation, this frequency was used to assess the effect of increasing doses of the drug. The effect of the stimulation was significantly increased by the lowest dose used of $0.05 \mathrm{mg} / \mathrm{kg}\left(\mathrm{S}_{2} / \mathrm{S}_{1}=1.4 \pm 0.1 ; n=5\right)$, was further increased by the $0.2 \mathrm{mg} / \mathrm{kg}$ dose $\left(\mathrm{S}_{2} / \mathrm{S}_{1}=2.0 \pm 0.5 ; n=5\right)$, and plateaued thereafter up until the highest dose administered of $3 \mathrm{mg} / \mathrm{kg}\left(\mathrm{S}_{2} / \mathrm{S}_{1}=1.8 \pm 0.4 ; n=5\right)$.

LSD was used in order to assess the effect of the activation of the terminal 5-HT autoreceptor by an exogenous agonist on the effectiveness of the electrical stimulation of the ascending 5 -HT pathway. The intravenous injection of $25 \mu \mathrm{g} / \mathrm{kg}$ of LSD failed to reduce the SII, value of CA3 pyramidal neurons produced by electrical stimulation of the afferent 5 -HT fibers at either 160 or $320 \mu \mathrm{A}$ (Fig. 6). Lower and higher doses (10-200 $\mu \mathrm{g} / \mathrm{kg}$ ) of LSD were subsequently administered at different times (1-5 min) prior to $S_{2}$. The mean SIL value was not reduced by these doses of LSD ( $n=5$; data not shown). To detect possible changes in postsynaptic hippocampal pyramidal neuron responsiveness to 5-HT or NE following acute intravenous administration of $\operatorname{LSD}(25 \mu \mathrm{g} / \mathrm{kg}), \mathrm{I} \cdot \mathrm{T}_{50}$ values and number of spikes suppressed per $\mathrm{nA}$ values for both monoamines were obtained before and after LSD administration. No alteration was observed (Table 2).

\section{Discussion}

The intravenous administration of methiothepin, without modifying the responsiveness of the hippocampal pyramidal neurons to microiontophoretically applied 5-HT, markedly increased the

Table 2. Responsiveness of CA3 hippocampal pyramidal neurons to microiontophoretic applications of 5-HT and NE before and after intravenous administration of LSD

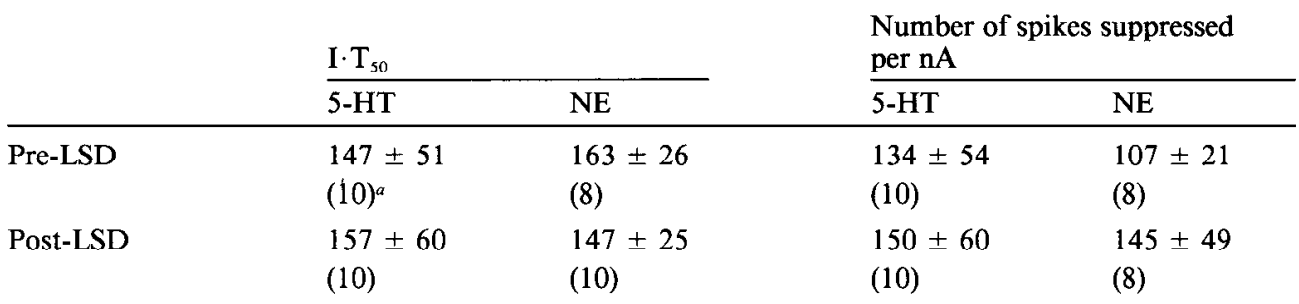

Values are expressed as means \pm SEM; responsiveness to $5-\mathrm{HT}$ and NE was assessed following a $25 \mu \mathrm{g} / \mathrm{kg}$ dose of LSD. Post-LSD values were not significantly different from pre-drug values (paired Student's $t$ test).

a Numbers in parentheses indicate the number of neurons tested. 
effect of the electrical activation of the ascending 5-HT axons on the firing activity of these same neurons. This suggests that methiothepin potentiates the efficacy of the 5-HT synapse by acting on the 5-HT neuron itself, presumably preventing activation of the terminal 5-HT autoreceptor by endogenously released 5-HT. Hence, the increased efficacy of the stimulation following methiothepin would reflect the cancellation of the autoregulatory effect of 5-HT released in the synaptic cleft. Such an interpretation is in keeping with the in vitro data showing that methiothepin increases ${ }^{3} \mathrm{H}-5-\mathrm{HT}$ release by a steric interaction with the terminal 5-HT autorcceptor (Ccrrito and Raitcri, 1979; Cox and Ennis, 1982; Farnebo and Hamberger, 1974; Göthert, 1980; Langer and Moret, 1982; Petitbone and Pflueger, 1984), as well as with the in vivo data of Baumann and Waldmeier (1984), who observed an increased 5-HIAA signal following intraperitoneal administration of methiothepin.

In addition to being a 5-HT autoreceptor antagonist, methiothepin is a catecholamine receptor antagonist (Keller et al., 1973; Lloyd and Bartholini, 1974; Monachon et al., 1972; Nelson et al., 1979; Zivkovic et al., 1975). This catecholamine receptor blocking property of methiothepin was confirmed here in vivo by the reduced responsiveness of CA3 hippocampal pyramidal neurons to microiontophoretically applied NE following its intravenous administration (Table 1). Catecholaminergic receptors may be present on the presynaptic 5-HT terminals (Baumann and Waldmeier, 1984; Frankhuyzen and Mulder, 1980; Galzin et al., 1984; Göthert and Huth, 1980). However, it is highly unlikely that this property of methiothepin could account for its potentiation of 5-H I synaptic transmission as neither adrenergic nor dopaminergic receptor blocking agents antagonize the effect of exogenous 5-HT on stimulus-evoked ${ }^{3} \mathrm{H}-5-\mathrm{HT}$ release from rat brain slices (Cox and Ennis, 1982; Langer and Moret, 1982), and as there is evidence that the catecholaminergic receptors on 5-HT terminals are not activated by endogenous catecholamines (Galzin et al., 1984).

Further evidence for the modulatory role of 5-HT terminal autoreceptors is provided by the present demonstration that altering the frequency of stimulation can modify its effectiveness. Increasing the frequency of stimulation from 0.8 to $5 \mathrm{~Hz}$ significantly decreased its effectiveness (Fig. 4). This finding, as well as its attenuation by the subsequent administration of a terminal 5-HT autoreceptor antagonist, is consistent with the notion that the neurotransmitter released by the stimulation activates the autoreceptor in an autoregulatory process. Presumably, at $5 \mathrm{~Hz}$, each pulse arrives at a time $(200 \mathrm{msec}$ after the preceding one) when the terminal 5-HT autoreceptors are still activated, releasing a smaller amount of the neurotransmitter. However, decreasing the frequency from 0.8 to $0.2 \mathrm{~Hz}$ only minimally increased the effect of the stimulation (Fig. 4). It is possible that the apparent inability of lowering the stimulus frequency to enhance its efficacy is due to the spontaneous firing of the 5-HT-containing neurons between stimulation pulses. The mean spontaneous firing rate of these neurons in chloral hydrate-anesthetized rats is of approximately $1.2 \mathrm{~Hz}$, the rate of discharge of most individual neurons varying between 0.5 and $3 \mathrm{~Hz}$ (Aghajanian, 1978; Blier and de Montigny, 1983). It is therefore possible that many 5-HT neurons "escaped" the lower frequency of stimulation, with the result that the impulse flow in 5-IIT axons might have been at a frequency closer to that of the spontaneous firing activity of the 5-HT neurons than to that experimentally used.

In our paradigm, LSD failed to reduce the synaptic efficacy of the stimulation of the ascending 5-HT pathway. The unchanged responsiveness of the postsynaptic neurons to $\mathrm{mi}$ croiontophoretically applied 5-HT (Table 2) rules out the possibility that a concomitant reduction of the sensitivity of the postsynaptic moiety accounts for the apparent lack of effect of LSD. The failure of LSD to modify the efficacy of the stimulation contrasts with the potent inhibitory effect of LSD in vitro on the stimulus-evoked ${ }^{3} \mathrm{H}-5-\mathrm{HT}$ release at the level of the terminal 5-HT autoreceptor (Farnebo and Hamberger, 1974; Katz and Kopin, 1969; Langer and Moret, 1982). However, these apparently conflicting results could be due to the fact that these in vitro experiments wcre carricd out in superfused slices, in which released 5-HT does not accumulate, thus enabling the autoreceptor to respond to an exogenous agonist. Consistent with such an explanation, Petitbone and Pflueger (1984), using an in vitro batch release system in which endogenously released 5-HT accumulates, failed to detect any influence of LSD on $\mathrm{K}^{+}$-evoked release of 5-HT. These authors postulated that, under their batch release conditions, endogenously released 5 -HT may be activating $5-\mathrm{HT}$ autoreceptors close to the saturation level so that the administration of an exogenous agonist has little effect. This interpretation is congruent with the present results, as well as with the in vivo results of Baumann and Waldmeier (1984), who found minimal alterations of the 5-HIAA signal in both the hypothalamus and hippocampus after systemic administration of LSD. Finally, the effectiveness of methiothepin in our model indicates that the autoreceptor is activated by endogenous 5-HT released by the stimulation, thus providing further support to this interpretation of the lack of effect of LSD.

This interpretation must be viewed with caution, however, in the light of the results of Wang and Aghajanian (1976), who used an in vivo electrophysiological technique whereby the response of postsynaptic amygdaloid neurons to the electrical stimulation of the afferent 5-HT axons was assessed. They reported a consistent reduction in the mean duration of suppression of firing activity following intravenous administration of $5-10 \mu \mathrm{g} / \mathrm{kg}$ of LSD. Although the authors proposed that this effect might have been due to an alteration of the properties of the axonal membrane at the level where the stimulation was applied, another plausible explanation is activation of the terminal 5-HT autoreceptor, resulting in a decreased release of 5-HT. That LSD may have different effects on the stimulation of the 5-HT pathway on amygdaloid and hippocampal neurons could be related to differing levels of 5-HT autoreceptor saturation in different rat CNS regions. This would be compatible with the varying potency of a same dose of methiothepin or ISD in altering 5-HT release from different rat CNS regions (Baumann and Waldmeier, 1984; Petitbone and Pflueger, 1984).

In conclusion, the present electrophysiological data support the modulatory role of the terminal 5-HT autoreceptor on 5-HT neurotransmission in vivo by demonstrating that endogenously released 5-HT activates the terminal 5-HT autoreceptor and that blockade of this activation by methiothepin results in an enhancement of 5-HT synaptic transmission.

\section{References}

Aghajanian, G. K. (1978) Feedback regulation of central monoaminergic neurons: Evidence from single cell recording studies. In Essays in Neurochemistry and Neuropharmacology, Vol. 3, M. B. H. Youdim, W. Lovenberg, D. F. Sharman, and J. R. Lagnado, eds., pp. 132, Wiley, London, UK.

Aghajanian, G. K., H. J. Haigler, and F. E. Bloom (1972) Lysergic acid diethylamide and serotonin: Direct action on serotonin-containing neurons in rat brain. Life Sci. $11: 615-622$.

Baumann, P. A., and P. C. Waldmeier (1981) Further evidence for negative feedback control of serotonin release in the central nervous system. Naunyn Schmiedebergs Arch. Pharmacol. 317: 36-43.

Baumann, P. A., and P. C. Waldmeier (1984) Negative feedback control of serotonin release in vivo: Comparison of 5-hydroxyindolacetic acid levels measured by voltammetry in conscious rats and by biochemical techniques. Neuroscience 11: 195-204.

Blier, P., and C. de Montigny (1983) Electrophysiological investigations on the effect of repeated zimelidine administration on serotonergic neurotransmission in the rat. J. Neurosci. 3: 1270-1278.

Cerrito, F., and M. Raiteri (1979) Serotonin release is modulated by presynaptic autoreceptors. Eur. J. Pharmacol. 57: 427-430. 
Chase, T. N., R. I. Katz, and I. J. Kopin (1969) Release of ${ }^{3}$ H-serotonin from brain slices. J. Neurochem. 16: 607-615.

Cox, B., and C. Ennis (1982) Characterization of 5-hydroxytryptaminergic autoreceptors in the rat hypothalamus. J. Pharm. Pharmacol. 34: 438-441.

Farnebo, L. O., and B. Hamberger (1971) Drug-induced changes in the release of ${ }^{3} \mathrm{H}$-monoamines from field stimulated rat brain slices. Acta Physiol. Scand. 371: 35-44.

Farnebo, I.. O., and B. Hamberger (1974) Regulation of ${ }^{3} \mathrm{H}-5$-hydroxytryptamine release from rat brain cortex slices. J. Pharm. Pharmacol. 26: 642-644.

Frankhuyzen, A. L., and $\Lambda$. H. Mulder (1980) Noradrenaline inhibits depolarization induced ${ }^{3} \mathrm{H}$-serotonin release from slices of rat hippocampus. Eur. J. Pharmacol. 63: 179-182.

Gallager, D. W., and G. K. Aghajanian (1975) Effects of chlorimipramine and lysergic acid diethylamide on efflux of precursor-formed ${ }^{3} \mathrm{H}$-serotonin: Correlations with serotonergic impulse flow. J. Pharmacol. Exp. Ther. 193: 785-795.

Galzin, A. M., C. Moret, and S. Z. Langer (1984) Evidence that exogenous but not endogenous norepinephrine activates the presynaptic alpha $_{2}$-adrenoceptors on serotonergic nerve endings in the rat hypothalamus. J. Pharmacol. Exp. Ther. 228: 725-732.

Göthert, M. (1980) Serotonin-receptor-mediated modulation of $\mathrm{Ca}^{2+}$ dependent 5-hydroxytryptamine release from neurones of the rat brain cortex. Naunyn Schmiedebergs Arch. Pharmacol. 314: 223-230.

Göthert, M., and H. Huth (1980) Alpha-adrenoceptor-mediated modulation of 5-hydroxytryptamine release from rat brain cortex slices. Naunyn Schmiedebergs Arch. Pharmacol. 313: 21-26.

Göthert, M., and G. Weinheimer (1979) Extracellular 5-hydroxytryptamine inhibits 5 -hydroxytryptamine release from rat brain cortex slices. Naunyn Schmiedebergs Arch. Pharmacol. 310: 93-96.

Haigler, H. J., and G. K. Aghajanian (1974) Lysergic acid diethylamide and serotonin: A comparison of effects on serotonergic neurons and neurons receiving a serotonergic input. J. Pharmacol. Exp. Ther. 188 . 688-699.

Hamon, M., S. Bourgoin, J. Jagger, and J. Glowinski (1974) Effects of lysergic acid diethylamide on synthesis and release of 5-HT in rat brain slices. Brain Res. 69: 265-280.

Kandel, E. R., and W. A. Spencer (1961) Electrophysiology of hippocampal neurons. II. After potential and repetitive firing. J. Neurophysiol. 24: 243-259.

Katz, R. I., and I. J. Kopin (1969) Effects of D-LSD and related compounds on release of norepinephrime- $\mathrm{H}^{3}$ and serotonin- $\mathrm{H}^{3}$ evoked from brain slices by electrical stimulation. Pharmacol. Res. Commun. 1: 54-62.

Keller, H. H., G. Bartholini, and A. Pletscher (1973) Increase of 3-methoxy-4-hydroxyphenylethylene glycol in rat brain by neuroleptic drugs. Eur. J. Pharmacol. 23: 183-186.

Langer, S. Z., and C. Moret (1982) Citalopram antagonizes the stim- ulation by lysergic acid diethylamide of presynaptic inhibitory serotonin autoreceptors in the rat hypothalamus. J. Pharmacol. Exp. Ther. 222: $220-226$.

Lloyd, K. G., and G. Bartholini (1974) The effect of methiothepin on cerebral monoamine neurons. Adv. Biochem. Psychopharmacol. 10: 305-309.

Martin, L. L., and E. Sanders-Bush (1982) Comparison of the pharmacological characteristics of $5-\mathrm{HT}_{1}$ and $5-\mathrm{HT}_{2}$ binding sites with those of serotonin autoreceptors which modulate serotonin release. Naunyn Schmiedebergs Arch. Pharmacol. 321: 165-170.

Monachon, M. A., W. P. Burkard, M. Jalfre, and W. Haefely (1972) Blockade of central 5-hydroxytryptamine receptors by methiothcpin. Naunyn Schmiedebergs Arch. Pharmacol. 274: 192-197.

de Montigny, C., and G. K. Aghajanian (1977) Preferential action of 5-methoxylryplamine and 5-methoxydimethyltryptamine on presynaptic serotonin receptors: A comparative iontophoretic study with LSD and 5-HT. Neuropharmacology 16: 811-818.

Moret, C. (1985) Pharmacology of the serotonin autoreceptor. In Neuropharmacology of Serotonin, A. R. Green, ed., pp. 21-49, Oxford U. P., Oxford, UK

Mounsey, I., K. A. Brady, J. Carroll, R. Fisher, and D. N. Middlemiss (1982) $\mathrm{K}^{+}$-evoked $\left[{ }^{3} \mathrm{H}\right]-5-\mathrm{HT}$ release from rat frontal cortex slices: The effect of 5-HT agonists and antagonists. Biochem. Pharmacol. 31: 49-53.

Nelson, D. L., A. Herbet, L. Pichat, J. Glowinski, and M. Hamon (1979) In vitro and in vivo disposition of ${ }^{3} \mathrm{H}$-methiothepin in brain tissues. Relationship to the effects of acute treatment with methiothepin on central serotoninergic receptors. Naunyn Schmiedebergs Arch. Pharmacol. 310: 25-33.

Paxinos, G., and C. Watson (1982) The Rat Brain in Stereotaxic Coordinates, Academic Press Australia, New South Wales, Australia.

Petitbone, D. J., and B. A. Ptlueger (1984) Effects of methiothepin and lysergic acid diethylamide on serotonin release in vitro and serotonin synthesis in vivo: Possible relation to serotonin autoreceptor function. J. Neurochem. 43: 83-90.

Rebec, G. V., S. D. Curtis, and K. S. Zimmerman (1982) Dorsal raphe neurons: Self-inhibition by an amphetamine-induced release of endogenous serotonin. Brain Res. 251: 374-379.

Wang, R. Y., and G. K. Aghajanian (1976) Inhibition of neurons in the amygdala by dorsal raphe stimulation: Mediation through a direct serotonergic pathway. Brain Res. 120: 85-102.

Wang, R. Y., C. de Montigny, B. I. Gold, R. H. Roth, and G. K. Aghajanian (1979) Denervation supersensitivity to serotonin in rat forebrain: Single cell studies. Brain Res. 178: 479-497.

Zivkovic, B., A. Guidotti, A. Revuelta, and E. Costa (1975) Effect of thioridazine, clozapine, and other antipsychotics on the kinetic state of tyrosine hydroxylase and on the turnover rate of dopamine in striatum and nucleus accumbens. J. Pharmacol. Exp. Ther. 194: 3746. 